\section{Rituximab for the treatment of allograft rejection in young renal transplant recipients}

Two studies have reported promising results on the use of rituximab for the treatment of graft rejection in pediatric and young adult renal transplant recipients.

Billing et al.'s pilot study investigated a regimen of high-dose intravenous immunoglobulin (IVIG) and rituximab for the treatment of chronic antibody-mediated rejection (CAMR). Their study included six patients aged 1026 years (including four men) who had received first renal transplants and developed CAMR according to the Banff 2005 criteria. Posttransplantation immunosuppressive therapy comprised a calcineurin inhibitor, mycophenolate mofetil and methylprednisolone. Median time from transplantation to diagnosis of CAMR was 44 months (range 6-120 months). On CAMR diagnosis, patients received four once-weekly doses of IVIG $(1 \mathrm{~g} / \mathrm{kg})$, and a single dose of rituximab $\left(375 \mathrm{mg} / \mathrm{m}^{2}\right) 1$ week after the final infusion of IVIG.

All patients had experienced decreases in glomerular filtration rate (GFR) during the 6 months before receiving the antirejection regimen of IVIG and rituximab. Following cessation of this treatment, GFR increased or stabilized in four patients. One patient experienced initial GFR stabilization, but lost the graft owing to progressive chronic rejection after 18 months; the other patient did not respond to therapy. At 12 months after treatment, mean GFR increase was 60\% (range $-48 \%$ to $+72 \%)$. The treatment regimen was well tolerated, with no treatment-specific adverse effects. The two patients who did not respond to the antirejection regimen were those whose index biopsies showed the most pronounced interstitial inflammation, most severe glomerulopathy and greatest deposition of $\mathrm{C} 4 \mathrm{~d}$ in peritubular capillaries.
The authors note that this finding might indicate that the antirejection regimen lacks the capacity to reverse advanced CAMR.

Zarkhin and colleagues investigated the safety and efficacy of rituximab for the treatment of biopsy-proven acute renal transplant rejection with B-cell infiltrates. Their open-label study randomly assigned 20 consecutive patients aged 2-23 years to standardcare rejection therapy (steroid pulsing and/or six doses of rabbit antithymocyte globulin $1.5 \mathrm{mg} / \mathrm{kg} ; n=10$ ) or to standard-care rejection therapy combined with rituximab (four onceweekly $375 \mathrm{mg} / \mathrm{m}^{2}$ infusions; $n=10$ ). Graft biopsies were performed at the time of rejection and 1 month and 6 months later; patients were monitored for 1 year.

Patient survival was $100 \%$ in both groups at 1 year. A trend towards better graft function, defined by creatinine clearance, was observed in the rituximab group compared with the control group throughout follow-up. A scoring system for acute rejection that ranged from 0 (no acute rejection) to 5 (Banff grade IIB rejection) was used to compare biopsies at 1 month and 6 months. Patients who received rituximab showed a significant improvement (decrease) in acute rejection scores on 1-month and 6-month biopsies $(P=0.0003$ and $P<0.0001$ compared with pretreatment scores, respectively). By contrast, the control patients' scores did not change significantly following treatment. At 6 months, acute rejection scores were significantly better (lower) in rituximab-treated patients than in control patients $(P=0.02)$. No serious adverse effects were reported and numbers of infectious complications were similar in the two groups.

\footnotetext{
Original articles Billing $\mathrm{H}$ et al. (2008) Successful treatment of chronic antibody-mediated rejection with IVIG and rituximab in pediatric renal transplant recipients. Transplantation 86: 1214-1221

Zarkhin V et al. (2008) A randomized, prospective trial of rituximab for acute rejection in pediatric renal transplantation. Am J Transplant 8: 2607-2617
} 